\title{
Identification of crucial microRNAs and genes in hypoxia-induced human lung adenocarcinoma cells
}

\author{
This article was published in the following Dove Press journal: \\ OncoTargets and Therapy \\ 25 July 2016 \\ Number of times this article has been viewed
}

\section{Ying Gengl,* \\ Lili Deng ${ }^{2, *}$ \\ Dongju Su' \\ Jinling Xiao' \\ Dongjie $\mathrm{Ge}^{3}$ \\ Yongxia $\mathrm{Bao}^{\prime}$ \\ Hui Jing ${ }^{4}$}

'Department of Respiratory, ${ }^{2}$ Department of Oncology, The Second Affiliated Hospital of Harbin Medical University, ${ }^{3}$ Department of Respiratory, The First Hospital of Harbin, ${ }^{4}$ Department of Emergency, The Second Affiliated Hospital of Harbin Medical University Harbin, Heilongjiang, People's Republic of China

*These authors contributed equally to this work
Correspondence: Yongxia Bao Department of Respiratory, The Second Affiliated Hospital of Harbin Medical University, Xuefu Road 246,

Harbin I5008I, Heilongjiang,

People's Republic of China

Tel/fax +86 45I 86605445

Email yongxiabao@hotmail.com

Hui Jing

Department of Emergency, The Second Affiliated Hospital of Harbin Medical

University, Harbin, Heilongjiang,

People's Republic of China

Tel/fax +86 45I 86296758

Email Jinghuil I7@I26.com
Background: Variations of microRNA (miRNA) expression profile in hypoxic lung cancer cells have not been studied so far. Therefore, using miRNA microarray technology, this study aimed to study the miRNA expression profile and investigate the potential crucial miRNAs and their target genes in hypoxia-induced human lung adenocarcinoma cells.

Materials and methods: Based on miRNA microarray, miRNA expression profiling of hypoxia-induced lung adenocarcinoma A549 cells was obtained. After identification of differentially expressed miRNAs (DE-miRNAs) in hypoxic cells, target genes of DE-miRNAs were predicted, and functional enrichment analysis of targets was conducted. Furthermore, the expression levels of DE-miRNAs and their target genes were validated by real-time quantitative polymerase chain reaction. In addition, using miRNA mimics, the effect of overexpressed DE-miRNAs on A549 cell behaviors (cell proliferation, cell cycle, and apoptosis) was evaluated.

Results: In total, 14 DE-miRNAs (nine upregulated miRNAs and five downregulated miRNAs) were identified in hypoxic cells, compared with normoxic cells. Target genes of both upregulated and downregulated miRNAs were enriched in the functions such as chromatin modification, and pathways such as Wnt signaling pathway and transforming growth factor (TGF)- $\beta$ signaling pathway. The expression levels of several miRNAs and their target genes were confirmed, including hsa-miR-301b/FOXF2, hsa-miR-148b-3p/WNT10B, hsa-miR-769-5p/(SMAD2, $A R I D 1 A$ ), and hsa-miR-622. Among them, hsa-miR-301b was verified to regulate FOXF2, and hsa-miR-769-5p was verified to modulate $A R I D 1 A$. In addition, the overexpression of hsamiR-301b and hsa-miR-769-5p significantly affected the cell cycle of A549 cells, but not cell proliferation and apoptosis.

Conclusion: miRNA expression profile was changed in hypoxia-induced lung cancer cells. Those validated miRNAs and genes may play crucial roles in the response of lung cancer cells to hypoxia.

Keywords: hypoxia, lung cancer, A549, microarray, hsa-miR-301b, hsa-miR-769-5p

\section{Introduction}

Lung cancer is a major cause of death worldwide, with 221,200 estimated deaths and 158,040 estimated new cases in the US in $2015 .{ }^{1}$ Lung adenocarcinoma is the most common subtype and accounts for $\sim 50 \%$ of lung cancer cases. ${ }^{2}$ Despite improvement in medical technology, the mortality of lung cancer is still high. ${ }^{3}$

Hypoxia is a common feature in almost all of the solid tumors, ${ }^{4}$ and it plays a significant role in the progress of tumors. ${ }^{5}$ In the past years, the molecular variations in hypoxic lung cancer cells have been discovered. In hypoxic lung cancer cells, the truncated voltage-dependent anion channel 1 (VDAC1- $\Delta \mathrm{C}$ ) associated with chemoresistance is expressed, which protects cancer cells from apoptosis, ${ }^{6}$ and $V D A C 1$ has been identified as a potential mitochondrial marker for cancer therapy. ${ }^{7}$ Furthermore, the expression of 
CXCR4 is dramatically enhanced in hypoxic non-small-cell lung cancer, which is modulated by the activation of hypoxiainducible factor (HIF)- $1 \alpha$ and $P I 3 K / P T E N / A K T / m T O R$ signal transduction pathway. ${ }^{8}$

Except for variations of protein expression, microRNAs (miRNAs) that play critical roles in the coordination of various cellular processes have also been discovered to express abnormally in hypoxic lung cancer cells. Previous studies have found that both miR-155 and miR-210 are highly expressed in hypoxic lung cancer cells, which radioprotect lung cancer cells. ${ }^{9,10}$ However, there is no study to explore the variation of miRNA expression profile in hypoxic lung cancer cells so far, and the regulatory relationships between varied miRNAs and genes in hypoxic lung cancer are still elusive.

In this study, to investigate the differentially expressed miRNAs (DE-miRNAs) in hypoxic lung cancer cells, miRNA expression profile analysis of hypoxia-induced lung adenocarcinoma A549 cells was performed based on miRNA microarray, which is a high-throughput technology to analyze miRNA expression profile. ${ }^{11}$ Furthermore, to investigate the potential DE-miRNAs-related functions in hypoxic cells, target genes of DE-miRNAs were predicted and functional enrichment analysis of targets was conducted. In addition, expression levels of DE-miRNAs and their target genes were validated by real-time quantitative polymerase chain reaction (RT-qPCR), and the effect of DE-miRNAs on A549 cell behaviors (cell proliferation, cell cycle, and apoptosis) was evaluated. These results may contribute to the study of miRNA expression profile in hypoxia-induced lung cancer cells, and the validated miRNAs and genes may be candidate biomarkers in the clinical therapy of lung cancer.

\section{Materials and methods Cell culture and establishment of hypoxic cell model}

Because a cell line was utilized in this study, the ethical permission obtained from a review board was unnecessary, as confirmed by the Ethic Committee for Use of Human Samples of the Harbin Medical University. The lung adenocarcinoma A549 cell line purchased from the Shanghai Cell Bank, Chinese Science Academy (Shanghai, People's Republic of China) was used in this study. The cells were divided into two groups: one group was cultured in a Biospherix $\mathrm{C} 21$ incubator $\left(1 \% \mathrm{O}_{2}\right.$, ProOx C21; Biospherix, $\mathrm{NY}, \mathrm{USA}$ ) at $37^{\circ} \mathrm{C}$ for 8 hours (hypoxia group) and another group was cultured in a normoxia condition at $37^{\circ} \mathrm{C}$ in $5 \%$ $\mathrm{CO}_{2}$ for 8 hours (normoxia group). Two group cells were cultured in a 1:1 mixture of Dulbecco's Modified Eagle's
Medium (DMEM) and Ham F12-medium (DMEM-F12) supplemented with $10 \%$ fetal bovine serum and $100 \mu \mathrm{g} / \mathrm{mL}$ penicillin-streptomycin (1:1). Once cells had reached confluence, they were subcultured with $0.25 \%$ Trypsinethylenediaminetetraacetic acid.

\section{RNA isolation, purification, and array procedures}

The total RNA for miRNA microarray preparation was isolated using mirVana ${ }^{\mathrm{TM}}$ miRNA Isolation Kit (Ambion, Austin, TX, USA), and then purified by miRNeasy Mini Kit (Qiagen, Hilden, Germany). The integrity of RNA was inspected by Agilent Bioanalyzer 2100 (Agilent Technologies, Santa Clara, CA, USA).

The RNA was reverse transcribed into cDNA after purification. Afterward, according to the manufacturer's instructions, the cDNA was labeled and homogenized. The labeling was performed by miRNA Complete Labeling and Hyb Kit (Agilent Technologies), and hybridization was performed on the human miRNA $(8 * 60 \mathrm{~K})$ v19.0 array in a hybridization oven (Agilent Technologies) at $55^{\circ} \mathrm{C}$ for 20 hours. Subsequently, slides were washed using Gene Expression Wash Buffer Kit (Agilent Technologies). Then, slides were scanned by Feature Extraction software 10.7 (Agilent Technologies) and Agilent Microarray Scanner (Agilent Technologies). Raw microarray data were normalized by a quantile algorithm in Gene Spring Software 11.0 (Agilent Technologies).

\section{Identification of DE-miRNAs}

The fold change (FC) model ${ }^{12}$ was used to identify miRNAs that were differentially expressed between hypoxia group and normoxia group. The FC value of miRNA expression between two groups was calculated, and miRNAs with FC value $>2$ were selected as DE-miRNAs.

\section{Prediction of DE-miRNA target genes}

Target genes of DE-miRNAs were predicted based on the TargetScan database, ${ }^{13}$ which is a commonly used tool for the prediction of miRNA targets by searching the presence of conserved sites ( $7 \mathrm{mer}$ and $8 \mathrm{mer}$ ) in the seed region of each miRNA. ${ }^{13}$

\section{Enrichment analysis of DE-miRNA targets}

The gene ontology (GO) database ${ }^{14}$ and Kyoto Encyclopedia of Genes and Genomes (KEGG) PATHWAY database ${ }^{15}$ were used to perform the GO functional and pathway enrichment analyses, respectively, of DE-miRNA target genes. 
The $P$-value of each term was calculated by Fisher's exact test ${ }^{16}$ and adjusted using the Benjamini-Hochberg method. ${ }^{17}$ Only the functional terms with adjusted $P$-value $<0.05$ were considered significant.

\section{RT-qPCR assay}

The total RNA for mRNA expression detection was extracted from A549 cells by TriZol reagent (Thermo Fisher Scientific, Waltham, MA, USA) according to the manufacturer's protocol. Afterward, total RNA was reverse transcribed using Moloney murine leukemia virus reverse transcriptase (Promega Corporation, Fitchburg, WI, USA). After cDNA synthesis, mRNA expression levels were tested using SYBR green qPCR supermix (Bio-Rad Laboratories Inc., Hercules, CA, USA). Primer sequences of genes are listed in Table $\mathrm{S} 1$.

Furthermore, the total RNA for miRNA expression detection was isolated using mirVana ${ }^{\mathrm{TM}}$ miRNA Isolation Kit and then purified by miRNeasy Mini Kit. miRNA expression levels were determined using miRcute miRNA qPCR Detection Kit (Tiangen, Beijing, People's Republic of China). Primer sequences of miRNAs are listed in Table S2. Expression levels of both mRNA and miRNA were analyzed by a CFX96 ${ }^{\mathrm{TM}}$ real-time detection system (Bio-Rad Laboratories Inc.). Relative gene expression was calculated using the $2^{-\Delta \Delta \mathrm{Ct}}$ method, ${ }^{18}$ and relative FC of miRNAs was normalized to endogenous $18 \mathrm{~S}$ rRNA.

\section{Western blot analysis}

Approximately $1 \times 10^{7}$ A549 cells from hypoxia group and normoxia group were harvested and lysed in radioimmunoprecipitation assay lysis buffer (1\% Triton-100, 0.1\% sodium dodecyl sulfate [SDS], $1 \mathrm{mmol} / \mathrm{L}$ ethylenediaminetetraacetic acid, $150 \mathrm{mmol} / \mathrm{L} \mathrm{NaCl}$, and $10 \mathrm{mmol} / \mathrm{L}$ Tris- $\mathrm{HCl}, \mathrm{pH}$ 7.5) for 30 minutes in ice. Subsequently, the lysate was transferred to $1.5 \mathrm{~mL}$ Eppendorf tubes, and then centrifuged at 12,000 rpm for 30 minutes at $4^{\circ} \mathrm{C}$. The supernatant was transferred to a fresh tube and mixed with equal volumes of $2 \times$ SDS and boiled for 20 minutes. Afterward, an equal volume of sample (containing $50 \mu \mathrm{g}$ of protein) was fractionated by $10 \%$ SDSpolyacrylamide gels and transferred onto polyvinylidene fluoride membrane for 2 hours. After blocking nonspecific binding sites with $5 \%$ skimmed milk in $1 \times$ Tris-buffered saline containing $0.1 \%$ Tween-20 (TBST, $0.1 \%$ Tween 20 , $50 \mathrm{mmol} / \mathrm{L}$ of Tris, $150 \mathrm{mmol} / \mathrm{L}$ of $\mathrm{NaCl}, \mathrm{pH}$ 7.6) for 1 hour, membranes were probed with anti-HIF $\alpha$ antibody (dilution 1:1,000, product code: 3716; Cell Signaling Technology, Boston, MA, USA) at $4{ }^{\circ} \mathrm{C}$ overnight, washed five times with $1 \times$ TBST, and incubated with horseradish-peroxidaseconjugated secondary antibody (dilution 1:10,000, product code: 1464325; Hoffman-La Roche Ltd., Basel, Switzerland) for 1 hour at room temperature. The membrane was visualized with the enhanced chemiluminescence Western blotting analysis system (Santa Cruz Biotechnology Inc., Dallas, TX, USA). Then, the protein expression level was quantitated by image densitometry, and the ratio of HIF $\alpha /$ glyceraldehyde-3 -phosphate dehydrogenase signal was statistically analyzed, respectively.

\section{Luciferase assay}

Based on the $3^{\prime}$ untranslated region ( $3^{\prime}$ UTR) sequences of genes in the miRBase database ${ }^{19}$ and the University of California Santa Cruz (UCSC) database ${ }^{20}$, gene primers were designed using the Primer5 software (PRIMER-E Ltd, Plymouth, UK). Primer sequences of $3^{\prime}$ UTR in genes are listed in Table S3.

A549 cells were plated in a 12-well plate and transfected with a dual luciferase reporter plasmid containing the $3^{\prime}$ UTR sequence of miRNA target gene using Lipofectamine 2000 (Thermo Fisher Scientific). A control empty plasmid was utilized as a negative control for reporter plasmids. Subsequently, miRNA mimics or scrambled controlled miRNA mimics (Shanghai GenePharma Co., Ltd., Shanghai, People's Republic of China) were cotransfected with cells. After transfection for 30 hours, Dual-Luciferase ${ }^{\circledR}$ Reporter Assay (product code: E1910; Promega Corporation) was performed according to the manufacturer's instructions. The "Renilla" luciferase activity was detected using a FLUOstar OPTIMA fluorescence microplate reader (BMG LABTECH, Offenburg, Germany) and normalized to the firefly luciferase activity.

\section{Methyl thiazolyl tetrazolium assay}

A549 cells were cotransfected with miRNA mimics or scrambled controlled miRNA mimics in a 96-well plate. After transfection for 24 hours, 48 hours, and 72 hours, methyl thiazolyl tetrazolium at $20 \mu \mathrm{L}$ was added to each well and incubated with cells for 4 hours. Following $200 \mu \mathrm{L}$ dimethyl sulfoxide being added in each well, the optical density was measured at $570 \mathrm{~nm}$ using a microplate reader (BioTek, Winooski, VT, USA).

\section{Flow cytometry analysis}

A549 cells cotransfected with miRNA mimics or scrambled controlled miRNA mimics for 72 hours were washed two times with precooled phosphate-buffered saline and then 
incubated with $20 \mu \mathrm{g} / \mathrm{mL}$ RNase A for 30 minutes at $37^{\circ} \mathrm{C}$, followed by staining with $10 \mathrm{mg} / \mathrm{mL}$ propidium iodide for 30 minutes in the dark at $37^{\circ} \mathrm{C}$. Afterward, the samples were evaluated by a flow cytometry (BD Biosciences, San Jose, CA, USA), and the data were analyzed using FlowJo software (Tree Star, San Carlos, CA, USA).

\section{Statistical methods}

Statistical analysis was performed using the SPSS 19.0 software (IBM Corporation, Armonk, NY, USA). One-way ANOVA followed by Duncan's test was used to compare the group means.

\section{Results}

\section{DE-miRNAs in hypoxic A549 cells}

Based on the Western blot analysis, HIF $\alpha$ was expressed in the A549 cells cultured in the hypoxia incubator $\left(1 \% \mathrm{O}_{2}\right)$, but not in cells cultured in the normoxic condition (Figure 1), indicating that the hypoxic A549 cell model was successfully established.

Based on the analysis of miRNA microarray data, a total of 14 miRNAs were differentially expressed between hypoxic cells and normoxic cells, including nine upregulated miRNAs

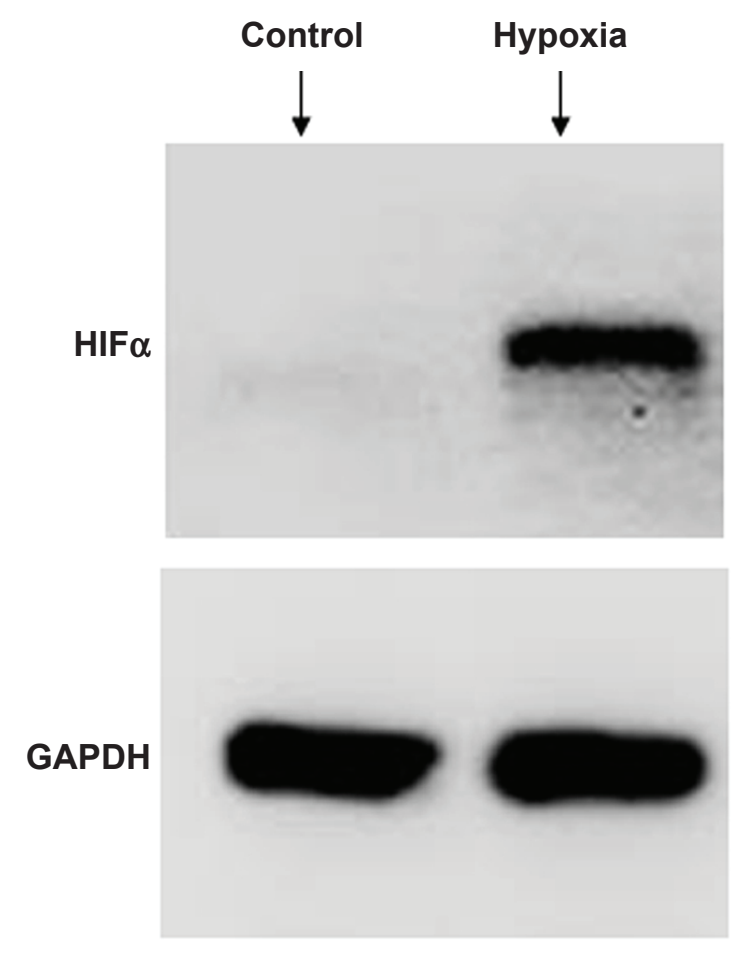

Figure I Expression of HIF $\alpha$ in A549 cells detected by Western blot analysis. Notes: "Control" represents the group of A549 cells cultured in the normoxic condition, and "hypoxia" represents the group of A549 cells cultured in a hypoxia incubator $\left(1 \% \mathrm{O}_{2}\right)$.

Abbreviations: HIF $\alpha$, hypoxia-inducible factor $\alpha$; GAPDH, glyceraldehyde-3phosphate dehydrogenase.
Table I DE-miRNAs in hypoxic A549 cells compared with normoxic cells

\begin{tabular}{lll}
\hline Category & Human miRNA name & FC \\
\hline Upregulated & hsa-miR-30Ib & 5.520343 \\
miRNAs & hsa-miR-148b-3p & 4.894202 \\
& hsa-miR-30d-3p & 4.533417 \\
& hsa-miR-769-5p & 4.265323 \\
& hsa-miR-190a & 4.106842 \\
& hsa-miR-II80 & 4.027596 \\
& hsa-miR-19b-I-5p & 3.745773 \\
& hsa-miR-596 & 3.717442 \\
Downregulated & hsa-miR-10b-3p & 3.554867 \\
miRNAs & hsa-miR-202-3p & -3.746923 \\
& hsa-miR-I255b-5p & -3.992389 \\
& hsa-miR-424-3p & $-4.0427 \mid 4$ \\
& hsa-miR-I8Ia-3p & -4.174865 \\
& hsa-miR-622 & -5.794464 \\
\hline
\end{tabular}

Abbreviations: DE, differentially expressed; FC, fold change; miRNA, microRNA.

(eg, hsa-miR-301b and hsa-miR-769-5p) and five downregulated miRNAs (eg, hsa-miR-622 and hsa-miR-181a-3p) in hypoxic cells (Table 1).

\section{Target genes of DE-miRNAs}

To investigate the genes that were regulated by the DEmiRNAs in hypoxic cells, target genes of DE-miRNAs were predicted using the TargetScan database. Four upregulated miRNAs (hsa-miR-301b, hsa-miR-148b-3p, hsa-miR769-5p, and hsa-miR-1180) and two downregulated miRNAs (hsa-miR-622 and hsa-miR-202-3p) were predicted to target a set of genes, eg, hsa-mir-301b regulated genes such as FOXF2 and CREB5; hsa-miR-769-5p modulated genes such as $A R I D 1 A$ and SMAD2; hsa-miR-622 regulated genes such as G3BP1 and CELF2; and hsa-miR-202-3p targeted genes such as TFAP2B and CLCN5 (Table 2).

\section{Functional analysis of DE-miRNAs targets}

To further reveal what biological functions the DE-miRNAs were involved in, the GO functional and pathway enrichment analyses of DE-miRNA target genes were performed. The targets of upregulated miRNAs were mainly enriched in the functions such as "transcription", "multicellular organismal development", "chromatin modification", "ion transport", and "cell cycle", which were also enriched by a set of target genes of downregulated miRNAs (Table 3).

Furthermore, a set of pathways was enriched by target genes of upregulated miRNAs such as "Wnt signaling pathway", "TGF- $\beta$ signaling pathway", "MAPK signaling pathway", and "focal adhesion". These pathways were also enriched by target genes of downregulated miRNAs (Table 4). 
Table 2 Target genes of DE-miRNAs

\begin{tabular}{lll}
\hline Category & Human miRNA name & Target genes \\
\hline Upregulated miRNAs & hsa-miR-30Ib & FOXF2, ESRI, MARK3, MYB, CREB5, etc \\
& hsa-miR-I48b-3p & NOG, WNTIOB, PTEN, SMAD5, SERBPI, etc \\
& hsa-miR-769-5p & ARIDIA, SMAD2, CPEBI, PTCHI, GRAMD3, etc \\
Downregulated miRNAs & hsa-miR-II80 & SCN5A, ISOC2, FOXP4, BAD, NRXN2, etc \\
& hsa-miR-622 & G3BPI, CELF2, SORBSI, SRSFI, CBX5, etc \\
& hsa-miR-202-3p & TFAP2B, CLCN5, ATP2B4, LIN28B, SNX30, etc \\
\hline
\end{tabular}

Abbreviations: DE, differentially expressed; miRNA, microRNA.

Table 3 The top ten enriched GO terms of target genes of upregulated and downregulated miRNAs

\begin{tabular}{|c|c|c|c|c|c|}
\hline Category & GO ID & GO name & $\begin{array}{l}\text { Adjusted } \\
P \text {-value }\end{array}$ & $\begin{array}{l}\text { Gene } \\
\text { count }\end{array}$ & Genes \\
\hline Target genes & GO:000635I & Transcription, DNA dependent & $6.22 \mathrm{E}-66$ & 6 & THRB, CREBI, NR3CI, ZNF2I I, AR, ESRI \\
\hline of upregulated & GO:0007275 & Multicellular organismal development & $2.36 \mathrm{E}-50$ & 180 & NDRG2, WNTIOB, BMP3, NUMBL, GAP43, etc \\
\hline \multirow{8}{*}{ miRNAs } & GO:00068II & lon transport & $1.68 \mathrm{E}-33$ & 70 & KCNQ4, KCNH8, ATP7A, KCNQ3, SLC24A4, etc \\
\hline & GO:0016568 & Chromatin modification & $|.23 \mathrm{E}-3|$ & 56 & DNMTI, SUV420HI, ARIDIA, SETD7, SUZI2, etc \\
\hline & GO:0007399 & Nervous system development & $6.69 \mathrm{E}-25$ & 92 & STAT3, GAP43, ATXN3, NOG, ARIDIA, etc \\
\hline & GO:0008I52 & Metabolic process & $3.97 E-24$ & 29 & RPPI 4, LPGATI, GFPTI, INSIGI, NATI 4, etc \\
\hline & GO:0010467 & Gene expression & 6.47E-20 & I & $E D A$ \\
\hline & GO:0007049 & Cell cycle & $5.98 \mathrm{E}-18$ & 86 & CD2AP, PAPD5, ERBB2IP, INGI, SETDB2, etc \\
\hline & GO:00064I2 & Translation & $6.00 \mathrm{E}-14$ & 7 & QRSLI, CYLD, AARSDI, MRPS25, EIF5A2, etc \\
\hline & GO:0006508 & Proteolysis & $1.56 \mathrm{E}-12$ & 39 & ADAMTS5, ADAMTS4, CTSA, RNPEPLI, LONRFI, etc \\
\hline Target genes of & GO:000635 I & Transcription, DNA dependent & $5.84 \mathrm{E}-35$ & 1 & $\mathrm{NR3Cl}$ \\
\hline downregulated & GO:00068II & Ion transport & 2.19E-22 & 44 & SLCO4CI, CACNAII, MRS2, ATP5FI, CACNG2, etc \\
\hline \multirow[t]{8}{*}{ miRNAs } & GO:0007275 & Multicellular organismal development & $5.2|\mathrm{E}-2|$ & 88 & EFNB3, WNT2B, LRPI, FLVCRI, DLL4, etc \\
\hline & GO:0016568 & Chromatin modification & $4.05 \mathrm{E}-13$ & 28 & ATXN7L3, TLK2, ING5, NCORI, EYA3, etc \\
\hline & GO:0007399 & Nervous system development & $2.48 \mathrm{E}-08$ & 42 & FGFII, TFAP2B, JHDMID, TMOD2, NLGNI, etc \\
\hline & GO:00064I7 & Regulation of translation & $7.58 \mathrm{E}-07$ & 16 & EIF2C3, IREB2, EIF2C4, CPEB2, DNAJCI, etc \\
\hline & GO:00064I2 & Translation & $9.56 \mathrm{E}-07$ & 3 & MRPS25, EIF5A, IGF2BP3 \\
\hline & GO:0007596 & Blood coagulation & $9.93 \mathrm{E}-07$ & I & ITGB3 \\
\hline & GO:0006333 & Chromatin assembly or disassembly & $1.91 \mathrm{E}-06$ & 6 & CDYL, MPHOSPH8, CHD9, CBX5, CHD7, ARID4A \\
\hline & GO:0008I52 & Metabolic process & $2.13 \mathrm{E}-06$ & 12 & AGPATI, IREB2, SMUGI, NAA30, RPPI 4, etc \\
\hline
\end{tabular}

Note: GO terms are ordered from small to large $P$-values.

Abbreviations: GO, gene ontology; miRNA, microRNA.

Table 4 The top ten enriched pathways of target genes of upregulated and downregulated miRNAs

\begin{tabular}{llllll}
\hline Category & Pathway & Pathway name & $\begin{array}{l}\text { Adjusted } \\
\text { P-value }\end{array}$ & $\begin{array}{l}\text { Gene } \\
\text { count }\end{array}$ & Genes \\
\hline ID & 4310 & Wnt signaling pathway & $2.60 \mathrm{E}-14$ & 47 & CCND3, SMAD2, FOSLI, CERI, WNTIOB, etc \\
of upregulated & 4144 & Endocytosis & $9.39 \mathrm{E}-13$ & 53 & SMAD2, FLTI, ADRBK2, PSD3, EPN2, etc \\
miRNAs & 4350 & TGF- $\beta$ signaling pathway & $6.16 \mathrm{E}-1 \mathrm{I}$ & 30 & SMAD2, TNF, NOG, ROCK2, BMPRIB, etc \\
& 4010 & MAPK signaling pathway & $7.26 \mathrm{E}-1 \mathrm{I}$ & 59 & FGFRI, RELA, FGF2, TNF, MAP3K5, etc \\
& 4916 & Melanogenesis & $2.96 \mathrm{E}-10$ & 32 & CALM2, CREB3L2, WNTIOB, KRAS, FZD6, etc \\
& 4510 & Focal adhesion & $6.59 \mathrm{E}-10$ & 47 & PAK6, FLTI, ITGA9, TNXB, XIAP, etc \\
& 4810 & Regulation of actin cytoskeleton & $1.46 \mathrm{E}-09$ & 48 & ARHGEF4, FGFRI, KRAS, FGF2, ARHGEF7, etc \\
& 4360 & Axon guidance & $2.49 \mathrm{E}-09$ & 35 & PAK6, DPYSL5, UNC5D, UNC5C, EPHB4, etc \\
& 4722 & Neurotrophin signaling pathway & $6.18 \mathrm{E}-09$ & 34 & KRAS, SH2B3, SOSI, MAP3K5, PIK3RI, etc \\
Target genes of & 4910 & Insulin signaling pathway & $1.56 \mathrm{E}-08$ & 35 & CALM2, KRAS, PRKACB, SOSI, PIK3RI, etc \\
downregulated & 4010 & MAPK signaling pathway & $6.97 \mathrm{E}-12$ & 43 & PLA2G3, TRAF6, MAP3KI, MEF2C, HRAS, etc \\
miRNAs & 4722 & Neurotrophin signaling pathway & $6.28 \mathrm{E}-09$ & 25 & MAP3KI, FOXO3, TRAF6, YWHAZ, FASLG, etc \\
& 4360 & Axon guidance & $3.77 \mathrm{E}-08$ & 24 & SRGAP3, SRGAPI, HRAS, NFAT5, ROBO2, etc \\
& $4 I 15$ & P53 signaling pathway & $5.46 \mathrm{E}-07$ & 16 & BBC3, IGFI, PMAIPI, CDK6, FAS, etc \\
& 4310 & Wnt signaling pathway & $5.7 I \mathrm{E}-07$ & 24 & WIFI, LRP6, DVL3, NFAT5, PPP2RIB, etc \\
& 4510 & Focal adhesion & $2.54 \mathrm{E}-06$ & 27 & LAMAI, COL4AI, IGFI, HRAS, COL3AI, etc \\
& 5220 & Chronic myeloid leukemia & $1.91 \mathrm{E}-04$ & 13 & PTPNII, CDK6, BCL2LI, RBI, TGFBRI, etc \\
& 4512 & ECM-receptor interaction & $2.16 \mathrm{E}-04$ & 14 & COL4A2, LAMAI, COL4AI, COL3AI, COL6A3, etc \\
& 4920 & Adipocytokine signaling pathway & $4.56 \mathrm{E}-04$ & 12 & PPARGCIA, CAMKKI, LEP, PPARA, ADIPOR2, etc \\
& 4350 & TGF- $\beta$ signaling pathway & $6.70 \mathrm{E}-04$ & 13 & PPP2RIB, TGFBRI, ACVRIC, ACVR2A, ACVR2B, etc \\
\hline
\end{tabular}

Note: Pathway terms are ordered from small to large $P$-values.

Abbreviations: ECM, extracellular matrix; MAPK, mitogen-activated protein kinase; miRNA, microRNA; TGF, transforming growth factor. 


\section{Expression of miRNAs and target genes in established hypoxic A549 cell model}

To verify the expression levels of DE-miRNAs and their targets in hypoxic A549 cells, RT-qPCR was utilized to determine the expression levels of three DE-miRNAs with a higher FC and a set of their targets (hsa-miR-301b/ [CREB5, FOXF2], hsa-miR-148b-3p/[NOG, WNT10B], hsamiR-769-5p/[SMAD2, ARID1A], hsa-miR-622/[G3BP1], and hsa-miR-202-3p/TFAP2B). According to the results of RT-qPCR, the downregulation of genes ARIDIA, FOXF2, $S M A D 2$, and $W N T 10 B$ was consistent with the results of bioinformatic analysis, whereas upregulated $C R E B 5$ and $N O G$, as well as downregulated $G 3 B P 1$ and $T F A P 2 B$, in hypoxic A549 cells was inconsistent with the predicted results (Figure 2A). Meanwhile, hsa-miR-301b, hsa-miR-148b-3p, and hsa-miR-769-5p were upregulated, and hsa-miR-622 was downregulated in hypoxic A549 cells, comparing with normoxic cells, which was consistent with the predicted results (Figure 2B).

\section{Regulatory relationships of DE-miRNAs and genes}

To further confirm the regulatory relationships of DEmiRNAs and their target genes, dual-luciferase reporter assay was performed. In this assay, the validated hsa-miR-301b and its target $F O X F 2$, as well as hsa-miR-769-5p and its target $A R I D 1 A$, were selected for the confirmation.

First, the cloning vectors containing the $3^{\prime}$ UTR sequence of FOXF2 and ARID1A was successfully established (Figure $3 \mathrm{~A}$ and Figure $\mathrm{S} 1$ ). According to the luciferase assay,
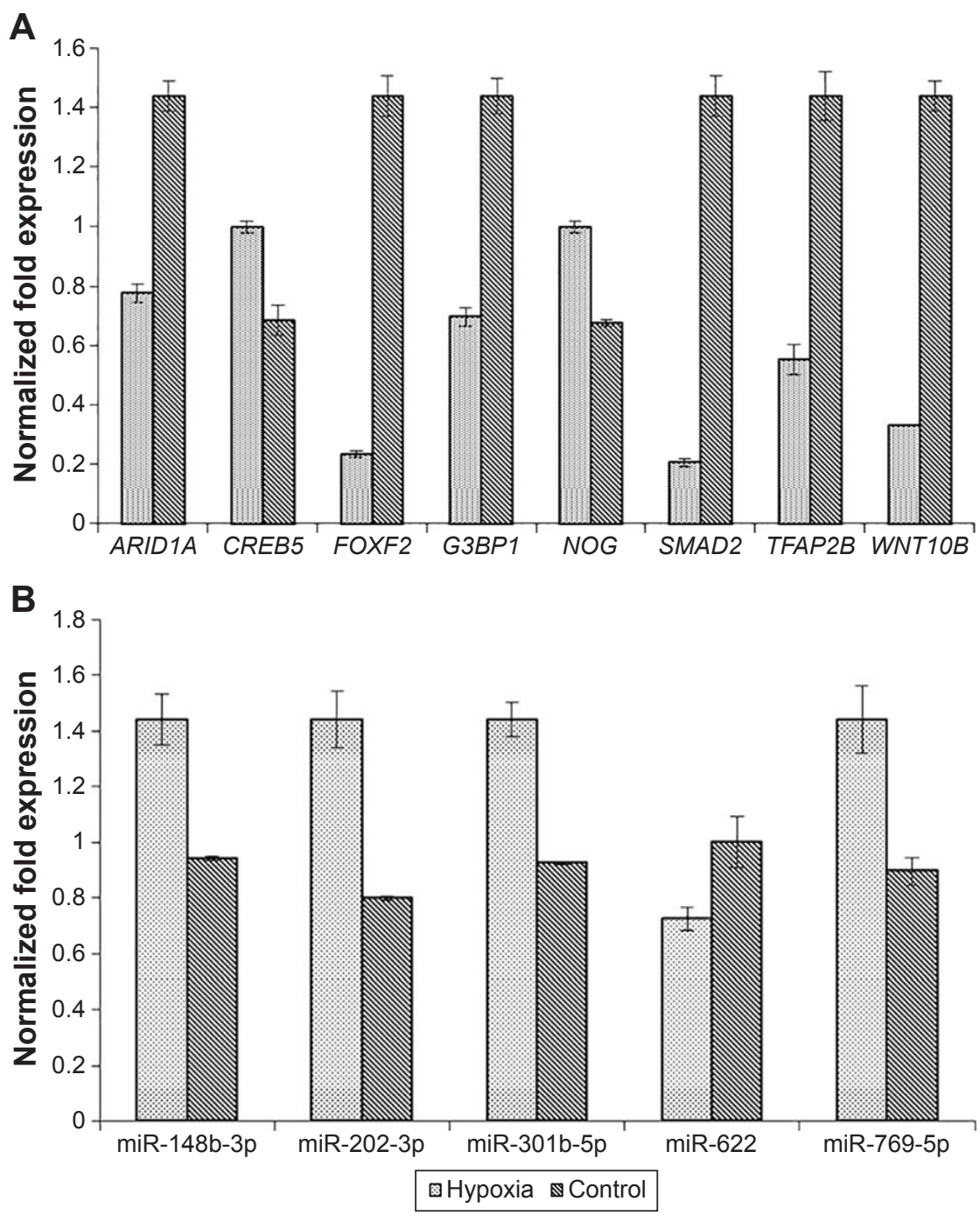

Figure 2 Expression levels of DE-miRNAs and target genes in hypoxic and normoxic A549 cells.

Notes: (A) Expression levels of miRNA target genes. (B) Expression levels of miRNAs. "Control" represents the group of A549 cells cultured in the normoxic condition, and "hypoxia" represents the group of $A 549$ cells cultured in a hypoxia incubator $\left(1 \% \mathrm{O}_{2}\right)$.

Abbreviations: DE, differentially expressed; miRNA, microRNA. 
A
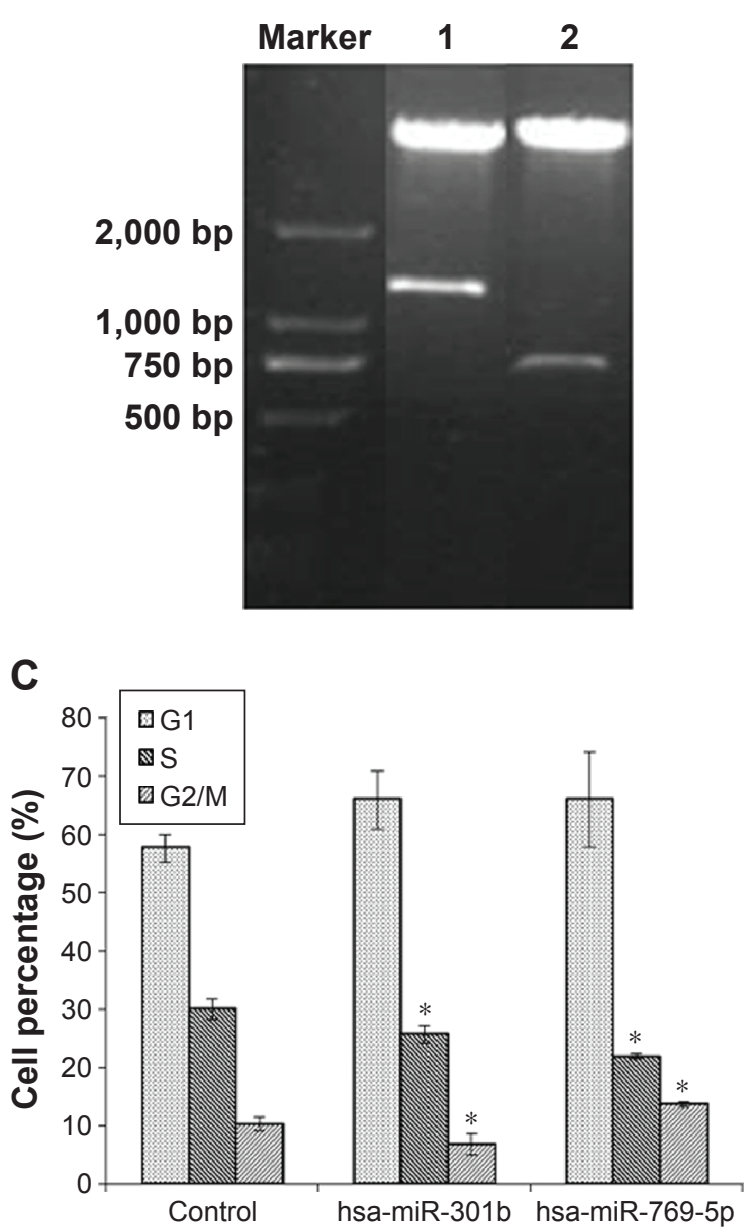

$\mathbf{E}$

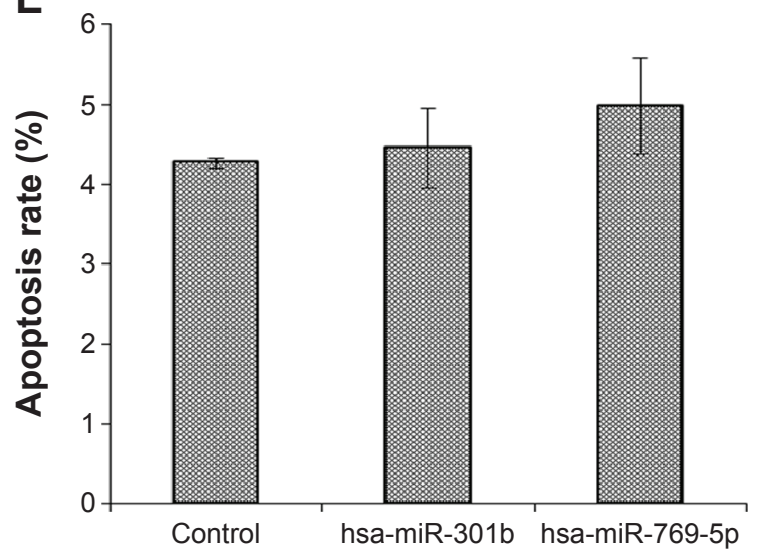

B

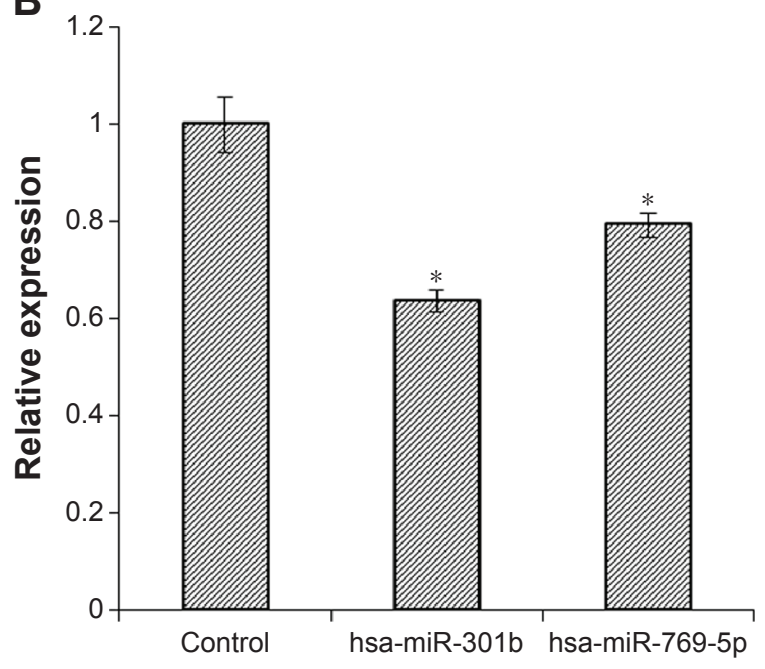

D

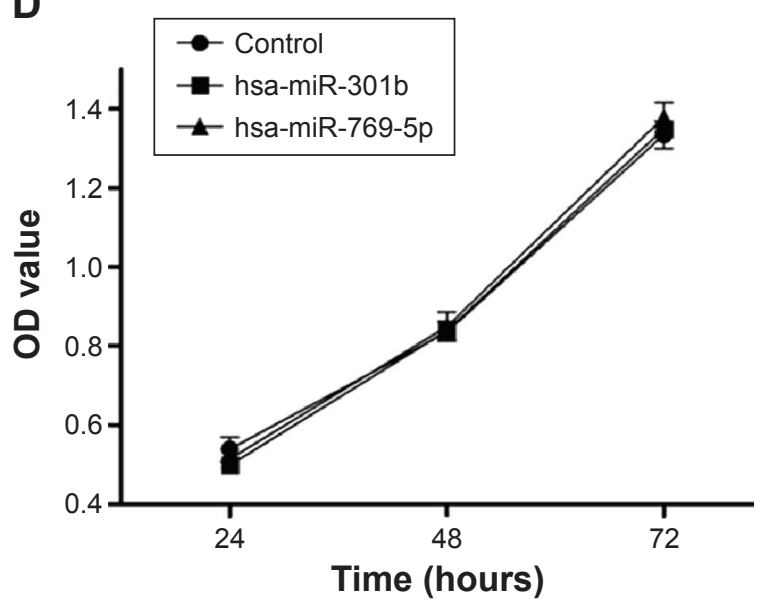

Figure 3 Validation of regulatory relationships of hsa-miR-30 Ib/hsa-miR-769-5p and their targets, and effect of miRNA overexpression on A549 cells.

Notes: (A) Electrophoretogram of enzyme-digested product of the cloning vector containing the $3^{\prime}$ UTR sequence of FOXF2 and/or ARIDIA. "I" represents the cloning vector containing the $3^{\prime}$ UTR sequence of ARIDIA and " 2 " represents the cloning vector containing the $3^{\prime}$ UTR sequence of FOXF2. (B) Luciferase activity of the 3 ' UTR sequence of FOXF2 and ARIDIA in A549 cells transfected by hsa-miR-30lb and hsa-miR-769-5p mimics. "Control" represents the group of A549 cells transfected by a scrambled miRNA sequence; "hsa-miR-30Ib" represents the group of A549 cells transfected by hsa-miR-30Ib mimic; "hsa-miR-769-5p" represents the group of A549 cells transfected by hsa-miR-769-5p mimic. (C) Cell percentage of A549 cells in the GI, S, and G2/M phases. "Control" represents the group of A549 cells transfected by a scrambled miRNA sequence; "hsa-miR-30Ib" represents the group of A549 cells transfected by hsa-miR-30Ib mimic; "hsa-miR-769-5p" represents the group of A549 cells transfected by hsa-miR-769-5p mimic. *P<0.05, compared with the control. (D) The mean OD value of A549 cells. "Control" represents the group of A549 cells transfected by a scrambled miRNA sequence; "hsa-miR-30Ib" represents the group of A549 cells transfected by hsa-miR-30Ib mimic; "hsa-miR-769-5p" represents the group of A549 cells transfected by hsa-miR-769-5p mimic. (E) Apoptosis rate of the A549 cells transfected by hsa-mir-30Ib and hsa-mir-769-5p mimics. "Control" represents the group of A549 cells transfected by a scrambled miRNA sequence; "hsa-miR-30lb" represents the group of A549 cells transfected by hsa-miR-30Ib mimic; "hsa-miR-769-5p" represents the group of $A 549$ cells transfected by hsa-miR-769-5p mimic.

Abbreviations: miRNA, microRNA; OD, optical density; UTR, untranslated region. 
the luciferase activity of the 3' UTR sequence of FOXF2 was significantly weaker in A549 cells transfected by hsamiR-301b mimic than the controls. Similarly, the luciferase activity of the 3' UTR sequence of ARIDIA was weaker in A549 cells transfected by hsa-miR-769-5p mimic than the controls (Figure 3B).

\section{Effect of DE-miRNAs on A549 cell behaviors}

To further investigate whether the overexpression of hsamiR-301b and hsa-miR-769-5p affected cell behaviors of A549 cells or not, cell proliferation, cell cycle, and apoptosis of A549 cells transfected by hsa-miR-301b and hsa-miR769-5p mimics were determined.

After transfection of miRNA mimics, according to the flow cytometry analysis, the percentage of A549 cells transfected by hsa-miR-301b and hsa-miR-769-5p mimics in the G1 phase was increasing, whereas the cell percentage was significantly reduced in the $\mathrm{S}$ phase, comparing with the controls (Figure 3C). However, the cell proliferation of A549 cells did not change significantly over time, comparing with the controls (Figure 3D). In addition, the apoptosis rate of A549 cells transfected by miRNA mimics did not significantly vary, comparing with the controls (Figure 3E).

\section{Discussion}

Hypoxia is common in almost all solid tumors, and it plays an important role in the development of tumors. ${ }^{4,5}$ In the current study, based on miRNA microarray, the miRNA expression profile in hypoxia-induced lung adenocarcinoma A549 cells was analyzed. In total, 14 DE-miRNAs (nine upregulated miRNAs and five downregulated miRNAs) were identified in hypoxic cells, comparing with normoxic cells. Among them, four upregulated miRNAs and two downregulated miRNAs were predicted to regulate a set of genes. The regulatory relationships of hsa-miR-301b and its target $F O X F 2$, as well as hsa-miR-769-5p and its target ARIDIA, were confirmed, and their expression levels in hypoxic A549 cells were also verified.

In this study, hsa-miR-301b was confirmed to be upregulated, and its target gene FOXF2 was downregulated in hypoxic A549 cells. The expression of miR-301b has been found to be increased in lung tissue of hypoxiatreated mice. ${ }^{21}$ In hypoxia-induced pulmonary hypertension, miR-130 promotes vasoconstriction by regulating the peroxisome proliferator-activated receptor $\gamma$ and vasoactive factors. ${ }^{22}$ A previous study has discovered the regulatory relationship of miR-301b and FOXF $2{ }^{23}$ FOXF2 encodes FOX F2, which is a transcription factor and is expressed in lung and placenta. ${ }^{24}$ In hypoxia-induced lung tissue, the DNA-binding activity of FoxF2 is significantly increased..$^{25}$ Besides, in lung tissue, Foxf2 mRNA exhibits a reduction after butylated hydroxytoluene injury. ${ }^{26}$ These results suggest the important role of FOXF2 in lung. Furthermore, in this study, the percentage of A549 cells transfected by hsamiR-301b mimics in the G1 phase was increasing, whereas the cell percentage was significantly reduced in the $\mathrm{S}$ and G2/M phases, compared with the controls, indicating that A549 cells were blocked in the G1 phase. However, the cell proliferation and apoptosis rate of A549 cells transfected by hsa-miR-301b mimics were not significantly affected. The results suggest that the overexpression of hsa-miR$301 \mathrm{~b}$ has no obvious influence on the cell proliferation and apoptosis of A549 cells. There is no other evidence to prove the significant roles of miR-301b and FOXF2 in hypoxiainduced lung cancer; thus, dysregulated hsa-miR-301b and FOXF2 may play pivotal roles in hypoxia-induced lung cancer cells via mediating transcription of other genes.

In the current study, hsa-miR-769-5p and its target ARID1A were also validated to be dysregulated in hypoxiainduced A549 cells. ARID1A encodes a protein belonging to the switch/sucrose non-fermentable (SWI/SNF) family; members of which have helicase and ATPase activities and modulate transcription of certain genes by changing the chromatin structure around those genes. ${ }^{27}$ In this study, ARID1A was distinctly enriched in the function of chromatin modification. A previous study has discovered that the SWI/ SNF chromatin-remodeling complex directly targets HIF $1 \alpha$ in the cellular response to hypoxia, and the damage of SWI/ SNF function makes cells resistant to hypoxia-induced cell cycle arrest. ${ }^{28}$ In this study, another target gene of hsa-miR769-5p, SMAD2, was also verified to be downregulated in hypoxic A549 cells. SMAD2 encodes a SMAD protein, which is a signal transducer that mediates multiple signaling pathways..$^{29}$ In this study, SMAD2 was enriched in the Wnt signaling pathway and TGF- $\beta$ signaling pathway. There is evidence that beta-catenin in the Wnt signaling pathway is able to enhance HIF1-mediated transcription, thus promoting cancer cell adaptation to hypoxia and survival..$^{30}$ Besides, HIF- $1 \alpha$ inactivates the Wnt signaling by binding to betacatenin, which contributes to the hypoxia-induced growth arrest of tumor cells. ${ }^{31}$ Furthermore, a study has reported that hypoxia enhances the expression of TGF- $\beta 1$ and the phosphorylation status of Smad2 in hepatic stellate cells. ${ }^{32}$ The mRNA expression level of TGF- $\beta$ receptor is distinctly 
increased in hypoxia-induced gastric cancer cells, and hypoxia stimulates the epithelial mesenchymal transition of gastric cancer cells via the TGF- $\beta /$ TGF- $\beta$ R signaling. ${ }^{33}$ These findings indicate the crucial roles of the Wnt signaling pathway and TGF- $\beta$ signaling pathway in the cellular response to hypoxia. Overall, hsa-miR-769-5p is likely to exhibit its functions in hypoxia-induced lung cancer cells via regulating the chromatin modification-related gene $A R I D 1 A$ and signaling pathway-related gene $S M A D 2$, which needs to be confirmed by further experiments.

Despite the aforementioned results, this study has some limitations. In this study, the effects of overexpression of DE-miRNAs cell behavior in the hypoxia condition have not been studied, which will be investigated in our further study. Besides, we would verify the expression of DE-miRNAs and their targets in lung adenocarcinoma tissue from patients in our future study.

\section{Conclusion}

A set of miRNAs and their target genes was validated to be differentially expressed in hypoxic lung cancer cells, compared with normoxic cells. Among them, hsa-miR-301b and its target $F O X F 2$, as well as hsa-miR-769-5p and its targets $A R I D 1 A$ and SMAD2, may play crucial roles in the response of lung cancer cells to hypoxia. These results provide new information for the study of hypoxia-induced lung cancer cells, and the DE-miRNAs and their targets are expected to be selected as candidate biomarkers for the clinical therapy of lung cancer.

\section{Acknowledgments}

This study was supported by the Natural Science Fund of Heilongjiang Province (No D201230).

\section{Disclosure}

The authors report no conflicts of interest in this work.

\section{References}

1. Siegel RL, Miller KD, Jemal A. Cancer statistics, 2015. CA Cancer J Clin. 2015;65(1):5-29.

2. Lortet-Tieulent J, Soerjomataram I, Ferlay J, Rutherford M, Weiderpass E, Bray F. International trends in lung cancer incidence by histological subtype: adenocarcinoma stabilizing in men but still increasing in women. Lung Cancer. 2014;84(1):13-22.

3. Nishino M, Klepeis VE, Yeap BY, et al. Histologic and cytomorphologic features of ALK-rearranged lung adenocarcinomas. Mod Pathol. 2012;25(11):1462-1472.

4. Talks KL, Turley H, Gatter KC, et al. The expression and distribution of the hypoxia-inducible factors HIF- $1 \alpha$ and HIF- $2 \alpha$ in normal human tissues, cancers, and tumor-associated macrophages. Am J Pathol. 2000; 157(2):411-421.
5. Dehdashti F, Mintun MA, Lewis JS, et al. In vivo assessment of tumor hypoxia in lung cancer with 60Cu-ATSM. Eur J Nucl Med Mol Imaging. 2003;30(6):844-850.

6. Brahimi-Horn MC, Ben-Hail D, Ilie M, et al. Expression of a truncated active form of VDAC1 in lung cancer associates with hypoxic cell survival and correlates with progression to chemotherapy resistance. Cancer Res. 2012;72(8):2140-2150.

7. Jia W, Zhang J-S, Jiang Y, Zheng Z-Y, Zhan X-B, Lin C-C. Structure of oligosaccharide F21 derived from exopolysaccharide WL-26 produced by Sphingomonas sp. ATCC 31555. Carbohydr Polym. 2012; 90(1):60-66.

8. Phillips RJ, Mestas J, Gharaee-Kermani M, et al. Epidermal growth factor and hypoxia-induced expression of CXC chemokine receptor 4 on non-small cell lung cancer cells is regulated by the phosphatidylinositol 3-kinase/PTEN/AKT/mammalian target of rapamycin signaling pathway and activation of hypoxia inducible factor-1 $\alpha . J$ Biol Chem. 2005; 280(23):22473-22481.

9. Babar IA, Czochor J, Steinmetz A, Weidhaas JB, Glazer PM, Slack FJ Inhibition of hypoxia-induced miR-155 radiosensitizes hypoxic lung cancer cells. Cancer Biol Ther. 2011;12(10):908-914.

10. Puissegur M, Mazure N, Bertero T, et al. miR-210 is overexpressed in late stages of lung cancer and mediates mitochondrial alterations associated with modulation of HIF-1 activity. Cell Death Differ. 2011;18(3): 465-478.

11. Liu X-G, Zhu W-Y, Huang Y-Y, et al. High expression of serum miR-21 and tumor miR-200c associated with poor prognosis in patients with lung cancer. Med Oncol. 2012;29(2):618-626.

12. Mutch DM, Berger A, Mansourian R, Rytz A, Roberts M-A. The limit fold change model: a practical approach for selecting differentially expressed genes from microarray data. BMC Bioinformatics. 2002;3:17.

13. Lewis BP, Shih IH, Jones-Rhoades MW, et al. Prediction of mammalian microRNA targets. Cell. 2003;115(7):787-798.

14. Gene Ontology Consortium. Gene ontology consortium: going forward. Nucleic Acids Res. 2015;43(Database issue):D1049-D1056.

15. Kanehisa M, Goto S. KEGG: Kyoto encyclopedia of genes and genomes. Nucleic Acids Res. 2000;28(1):27-30.

16. Jung SH. Stratified Fisher's exact test and its sample size calculation. Biom J. 2014;56(1):129-140.

17. Benjamini $Y$, Hochberg Y. Controlling the false discovery rate: a practical and powerful approach to multiple testing. J R Stat Soc Series B Stat Methodol. 1995;57(1):289-300.

18. Livak KJ, Schmittgen TD. Analysis of relative gene expression data using real-time quantitative PCR and the $2-\delta \delta \mathrm{ct}$ method. Methods. 2001;25(4):402-408.

19. Griffiths-Jones S. miRBase: microRNA sequences and annotation. Curr Protoc Bioinform. 2010;29:12.9.1-12.9.10.

20. Fujita PA, Rhead B, Zweig AS, et al. The UCSC genome browser database: update 2011. Nucleic Acids Res. 2010;39 (Database issue): D876-D882.

21. Brock M, Haider TJ, Vogel J, et al. The hypoxia-induced microRNA-130a controls pulmonary smooth muscle cell proliferation by directly targeting CDKN1a. Int J Biochem Cell Biol. 2015;61: 129-137.

22. Bertero T, Cottrill K, Krauszman A, et al. The microRNA-130/301 family controls vasoconstriction in pulmonary hypertension. $J$ Biol Chem. 2015;290(4):2069-2085.

23. Shi W, Gerster K, Alajez NM, et al. MicroRNA-301 mediates proliferation and invasion in human breast cancer. Cancer Res. 2011;71(8): 2926-2937.

24. Hellqvist M, Mahlapuu M, Samuelsson L, Enerback S, Carlsson P. Differential activation of lung-specific genes by two forkhead proteins, FREAC-1 and FREAC-2. J Biol Chem. 1996;271(8): 4482-4490.

25. Tharappel JC, Cholewa J, Espandiari P, Spear BT, Gairola CG, Glauert HP. Effects of cigarette smoke on the activation of oxidative stress-related transcription factors in female $\mathrm{A} / \mathrm{J}$ mouse lung. J Toxicol Environ Health A. 2010;73(19):1288-1297. 
26. Kalinichenko VV, Zhou Y, Shin B, et al. Wild-type levels of the mouse forkhead box f1 gene are essential for lung repair. Am J Physiol Lung Cell Mol Physiol. 2002;282(6):L1253-L1265.

27. Dallas PB, Pacchione S, Wilsker D, Bowrin V, Kobayashi R, Moran E. The human SWI-SNF complex protein p270 is an ARID family member with non-sequence-specific DNA binding activity. Mol Cell Biol. 2000; 20(9):3137-3146.

28. Kenneth NS, Mudie S, van Uden P, Rocha S. SWI/SNF regulates the cellular response to hypoxia. J Biol Chem. 2009;284(7):4123-4131.

29. Takenoshita S, Mogi A, Nagashima M, et al. Characterization of the MADH2/Smad2 gene, a human Mad homolog responsible for the transforming growth factor- $\beta$ and activin signal transduction pathway. Genomics. 1998;48(1):1-11.
30. Kaidi A, Williams AC, Paraskeva C. Interaction between $\beta$-catenin and HIF-1 promotes cellular adaptation to hypoxia. Nat Cell Biol. 2007;9(2):210-217.

31. Lim J-H, Chun Y-S, Park J-W. Hypoxia-inducible factor- $1 \alpha$ obstructs a Wnt signaling pathway by inhibiting the hARD1-mediated activation of $\beta$-catenin. Cancer Res. 2008;68(13):5177-5184.

32. Shi Y-F, Fong C-C, Zhang Q, et al. Hypoxia induces the activation of human hepatic stellate cells LX-2 through TGF- $\beta$ signaling pathway. FEBS Lett. 2007;581(2):203-210.

33. Matsuoka J, Yashiro M, Doi Y, et al. Hypoxia stimulates the EMT of gastric cancer cells through autocrine TGF $\beta$ signaling. PLoS One. 2013;8(5):e62310. 


\section{Supplementary materials}

Table SI Primer sequences of genes

\begin{tabular}{ll}
\hline Gene & Primer sequences \\
\hline FOXF2 & Forward: CCGTTACCAGCATCACTCTACT \\
CREB5 & Reverse: CGCAGGGCTTAATATCCTGACA \\
NOG & Forward: CCCTGCCCAACCCTACAATG \\
& Reverse: GGACCTTGCATCCCCATGAT \\
WNTIOB & Forward: CCATGCCGAGCGAGATCAAA \\
SMAD2 & Reverse: TCGGAAATGATGGGGTACTGG \\
& Forward: CATCCAGGCACGAATGCGA \\
G3BPI & Reverse: CGGTTGTGGGATCAATGAAGA \\
& Forward: TCATAGCTTGGATTTACAGCCAG \\
TFAP2B & Reverse: TTCTACCGTGGCATTTCGGTT \\
& Forward: CCAGCAGAACTCTCACGACC \\
GAPDH & Reverse: CTGAGCGAAGGACGAAGACG \\
& Forward: CCATCCCGGAATGGAAGACG \\
I8S rRNA & Reverse: TCACCGATTTGGGAGGAACTG \\
& Forward: GGAGCGAGATCCCTCCAAAAT \\
\hline
\end{tabular}

Table S2 Primer sequences of miRNAs

\begin{tabular}{ll}
\hline miRNA & Primer sequences \\
\hline miR-30lb-5p & GCTCTGACGAGGTTGCACTACT \\
miR-148b-3p & TCAGTGCATCACAGAACTTTGT \\
miR-769-5p & TGAGACCTCTGGGTTCTGAGCT \\
miR-622 & ACAGTCTGCTGAGGTTGGAGC \\
miR-202-3p & AGAGGTATAGGGCATGGGAA \\
U6-Forward & CTCGCTTCGGCAGCACA \\
U6-Reverse & AACGCTTCACGAATTTGCGT \\
\hline
\end{tabular}

Abbreviation: miRNA, microRNA.

Table S3 Primer sequences of $3^{\prime}$ UTR in genes FOXF2 and ARIDIA

\begin{tabular}{ll}
\hline Gene 3' UTR & Primer sequences \\
\hline FOXF2 3' UTR & EcoRI Forward: GGAATTCACGGAAAGAGGCCAAGCGATG \\
FOXF2 3' UTR & Xhol Reverse: CCGCTCGAGTGTTATATACATTTTATTGAAAAAA \\
ARIDIA 3' UTR & EcoRI Forward: GGAATTCCAGCCGTGGGACACCTCC \\
ARIDIA 3' UTR & Xhol Reverse: CCGCTCGAGAAAGTCTTACCAAGATTTAATGTAC
\end{tabular}

Abbreviation: UTR, untranslated region. 
A

FOXF2 3' UTR sequence from UCSC

ACGGAAAGAGGCCAAGCGATGGCCGCTCTCTCCTCTCCCCTCCTCAGAGGGGGCA GATAGAAACTGGGACGGATTCAAGTCACATGCACGCGGATAGCAGTAAGCCACACAC CTGCCACTTAGCCAGAATGCCCAGGATCGCGTTGGTCACTGTTATTTGCCTACTGCT GGAAGAAGGACAACCGCTGGCAAGGTAGCGTTCCCCAATCTGAATACCTGCAGGCT CCCACATGAGGGAGAGGGCAGACTCAGGTGGGAAGATGTGCCATGCGTAAGGCATC AACGTGTATCTGTGGGATCTTCGTTGCCTTCAGTAATCAGGGTGTGAAAAAAGCAGA CAAGTTGTGTGTGTGTGTGTGTGTCTAAGAAAACTTGTGTGCTTTTCAAAAAGGCAG TGCTAAGCACAAGATTTCAAGAAAGCCTCTTCTTGTTGCCTAGCTGAGTGGGAGAGT CATTTTCCCCAGACACTACATTTGGATACAGGTGCCAAAGAACATTATTAAGGAATTAT TTAGAAACAATGTGTCTAGTTTAAGAAAGTGGTTTTCAGTATTGTGACAATACAACGTT TTTACAAGGTTGTTTCTACCACCATATTTTAAAGATATTTTTATGACCGTGTATACTCA CACTTTGCTTGTATTTTAAAAGGAGGATATATTTGCACTTATGTATACTTTACAGTTTG CCAAAATATTTTGTTGTAAAATTTTTTTTCAATAAAATGTATATAACA

\section{C}

ARID1A 3' UTR sequence from UCSC

CAGCCGTGGGACACCTCCCCCCCCCGTGTGTGTGTGCGTGTGTGGAGAACTTAGAA ACTGACTGTTGCCCTTTATTTATGCAAAACCACCTCAGAATCCAGTTTACCCTGTGCT GTCCAGCTTCTCCCTTGGGAAAAAGTCTCTCCTGTTTCTCTCTCCTCCTTCCACCTC CCСTCCСTCCATCACCTCACGCCTTTCTGTTCCTTGTCCTCACCTTACTCCCCTCAG GACCCTACCCCACCCTCTTTGAAAAGACAAAGCTCTGCCTACATAGAAGACTTTTTTT ATTTTAACCAAAGTTACTGTTGTTTACAGTGAGTTTGGGGAAAAAAAATAAAATAAAAA TGGCTTTCCCAGTCCTTGCATCAACGGGATGCCACATTTCATAACTGTTTTTAATGGT AAAAAAAAAAAAAAAAAATACAAAAAAAAATTCTGAAGGACAAAAAAGGTGACTGCTG AACTGTGTGTGGTTTATTGTTGTACATTCACAATCTTGCAGGAGCCAAGAAGTTCGCA GITGTGAACAGACCCTGTTCACTGGAGAGGCCTGTGCAGTAGAGTGTAGACCCTTT CATGTACTGTACTGTACACCTGATACTGTAAACATACTGTAATAATAATGTCTCACATGG AAACAGAAAACGCTGGGTCAGCAGCAAGCTGTAGTTTTTAAAAATGTTTTTAGTTAAA CGTTGAGGAGAAAAAAAAAAAAGGCTTTTCCCCCAAAGTATCATGTGTGAACCTACAA CACCCTGACCTCTTTCTCTCCTCCTTGATTGTATGAATAACCCTGAGATCACCTCTTA GAACTGGTTTTAACCTTTAGCTGCAGCGGCTACGCTGCCACGTGTGTATATATATGAC GTTGTACATTGCACATACCCTTGGATCCCCACAGTTTGGTCCTCCTCCCAGCTACCC CTTTATAGTATGACGAGTTAACAAGTTGGTGACCTGCACAAAGCGAGACACAGCTATT TAATCTCTTGGCAGATATCGCCCCTCTTGGTGCGATGCTGTACAG GTCTCTGTAAAAA GTCCTTGCTGTCTCAGCAGCCAATCAACTTATAGTTTATTTTTTCTGGGTTTTTGTTT TGTTTTGTTTTCTTTCTAATCGAGGTGTGAAAAAGTTCTAGGTTCAGTTGAAGTTCTGA TGAAGAAACACAATTGAGATTTTTTCAGTGATAAAATCTGCATATTTGTATTTCAACAAT GTAGCTAAAACTTGATGTAAATTCCTCC TITTCC TT TTGGCTTAATGAATATCAT ITATTCAGTATGAAATCTTTATACTATATGTTCCACGTGTTAAGAATAAATGTACATTAAA TCTTGGTAAGACTTT
B

pCDNA3 Luc-FOXF2 3' UTR sequence results

GGGGGGATCC(ECOR1)GAATTCACGGAAAGAGGCCAAGCGATGGCCGCTCTCTCC TCTCCCCTCCTCAGAGGGGGCAGATAGAAACTGGGACGGATTCAAGTCACATGCAC GCGGATAGCAGTAAGCCACACACCTGCCACTTAGCCAGAATGCCCAGGATCGCGTT GGTCACTGTTATTTGCCTACTGCTGGAAGAAGGACAACCGCTGGCAAGGTAGCGTTC CCCAATCTGAATACCTGCAGGCTCCCACATGAGGGAGAGGGCAGACTCAGGTGGGA AGATGTGCCATGCGTAAGGCATCAACGTGTATCTGTGGGATCTTCGTTGCCTCAGTA ATCAGGGTGTGAAAAAAGCAGACAAGTTGTGTGTGTGTGTGTGTGTCTAAGAAAACT TGTGTGCTTTTCAAAAAGGCAGTGCTAAGCACAAGATTTCAAGAAAGCCTCTTCTTGT TGCCTAGCTGAGTGGGAGAGTCATTTTCCCCAGACACTACATTTGGATACAGGTGCC AAAGAACATTATTAAGGAATTATTTAGAAACAATGTGTCTAGTTTAAGAAAGTGGTTTTC AGTATTGTGACAATACAACGTTTTTACAAGGTTGTTTCTACCACCATATTTTAAAGATA TTTTIATGACCGTGTATACTCACACTTTGCTTGTATTTTAAAGGAGGATATATTTGCAC ITATGTATACTTTACAGTTGCCAAATATTTT GTTGTAAAATTTTTTCAATAAAATGTA TATAACACTCGAG(Xh01)CATGCATCTAGAGGGCCCTATTCTATAGTGTCACCTAAATG CTAGAGCTCGCTGATCAGCCTCGACTGTGCCTTCTAGTTGCCAGCCATCTGTTGTTT GCCCCTCCCCCGTGCCTTCCTTGACCCTGGAAGGTGCCACTCCCACTGTCCTTTCC TAATAAAATGAGGAAATTGCATCGCATTGTCTGAGTAGGTGTCATTCTATTCTGGGGG GTGGGGTGGGGCAGGACAGCAGGGGGAG

D

PCDNA3 Luc-ARID1A 3' UTR sequence

GGGACC(ECOR1)GAATTCCAGCCGTGGGACACCTCCCCCCCCCGTGTGTGTGTGCG TGTGTGGAGAACTTAGAAACTGACTGTTGCCCTTTATTTATGCAAAACCACCTCAGAA TCCAGTTTACCCTGTGCTGTCCAGCTTCTCCCTTGGGAAAAAGTCTCTCCTGTTTCT СTCTCCTCCTTCСАССTCСССTCССTCСATCACCTCACGCCTTTCTGTTCCTTGTCC CACCTTACTCCCCTCAGGACCCTACCCCACCCTCTITGAAAAGACAAAGCTCTGCCT ACATAGAAGACTTTTTTTATTTTAACCAAAGTTACTGTTGTTTACAGTGAGTTTGGGGA AAAAAAATAAAAATAAAAATGGCTTTCCCAGTCCTTGCATCAACGGGATGCCACATTTCA TAACTGTTTTTAATGGTAAAAAAAAAAAAAAAAATACAAAAAAAAATTCTGAAGGACAA AAAAGGTGACTGCTGAACTGTGTGTGGTTTATTGTTGTACATTCACAATCTTGCAGGA GCCAAGAAGTTCGCAGTTGTGAACAGACCCTGTTCACTGGAGAGGCCTGTGCAGTA GAGTGTAGACCCTTTCATGTACTGTACTGTACACCTGATACTGTAAACATACTGTAATA ATAATGTCTCACATGGAAACAGAAAACGCTGGGTCAGCAGCAAGCTGTAGTTTTTAAA AATGTTTTTAGTTAAACGTTGAGGAGAAAAAAAAAAAAGGCTTTTCCCCCAAAGTATC ATGTGTGAACCTACAACACCCTGACCTCTTTCTCTCCTCCTTGATTGTATGAATAACC CTGAGATCACCTCTTAGAACTGGTTTTAACCTTTAGCTGCAGCGGCTACGCTGCCAC GTGTGTATATATATGACGTTGTACATTGCACATACCCTTGGATCCCCACAGTTTGGTCC TCCTCCCAGCTACCCCTTTATAGTATGACGAGITAACAAGTTGGTGACCTGCACAAAG CGAGACACAGCTATTTAATCTCTTGCCAGATATCGCCCCTCTTGGTGCGATGCTGTAC AGGTCTCTGTAAAAAGTCCTTGCTGTCTCAGCAGCCAATCAACTTATAGTTTATTTTTT TCTGGGTTTTTGTTTTGTTTTGTTTTCTTCTAATCGAGGTGTGAAAAAGTTCTAGGTT CAGTTGAAGTTCTGATGAAGAAACACAATTGAGATTTTTTCAGTGATAAAATCTGCATA TTTGTATTTCAACAATGTAGCTAAAACTTGATGTAAATTCCTCC TITTTCCTTITTC GCTTAATGAATATCATTTATTCAGTATGAAATCTTTATACTATATGTTCCACGTGTTAAGA ATAAATGTACATTAAATCTTGGTAAGACTTTCTCGAG(Xho1)CATGCATCTAGAGGGCC CTATTCTATAGTGTCACCTAAATGCTAGAGCTCGCTGATCAGCCTCGACTGTGCCTTC TAGTTGCCAGCCATCTGTTGTTTGCCCCTCCCCCGTGCCTTCCTTGACCCTGGAAGG TGCCACTCCCACTGTCCTTTCCTAATAAAATGAGGAAATTGCATCGCATTGTCTGAGT AGGTGTCATTCTATTCTGGGGGGTGGGGG

Figure SI 3' UTR sequences of genes ARIDIA and FOXF2.

Notes: (A) 3' UTR sequence of FOXF2 gene in the UCSC database. (B) 3' UTR sequence of FOXF2 gene in the PCDNA3 vector. (C) 3' UTR sequence of ARIDIA gene in the UCSC database. (D) 3' UTR sequence of ARIDIA gene in the PCDNA3 vector.

Abbreviations: UCSC, University of California Santa Cruz; UTR, untranslated region.

OncoTargets and Therapy

\section{Publish your work in this journal}

OncoTargets and Therapy is an international, peer-reviewed, open access journal focusing on the pathological basis of all cancers, potential targets for therapy and treatment protocols employed to improve the management of cancer patients. The journal also focuses on the impact of management programs and new therapeutic agents and protocols on

\section{Dovepress}

patient perspectives such as quality of life, adherence and satisfaction The manuscript management system is completely online and includes a very quick and fair peer-review system, which is all easy to use. Visit http://www.dovepress.com/testimonials.php to read real quotes from published authors. 